\title{
A Generalization of Cauchy-Bunyakovsky Integral Inequality Via Means with Max and Min Values
}

\author{
P. Agarwal, A. A. Korenovskii and S. M. Sitnik
}

\begin{abstract}
In the paper, we give a brief survey of a method for constructing generalizations of Cauchy-Bunyakovsky integral inequality using abstract mean values. One special inequality of this type is considered in details in terms of min and max functions. Some direct proofs of this inequality are given and application to inequalities for special functions. Also related recent references are briefly considered.
\end{abstract}

\section{Means and Generalizations of Cauchy-Bunyakovsky Integral Inequality}

\subsection{Introduction}

Cauchy-Bunyakovsky inequalities for finite sums, series and integrals are among the most important inequalities with many applications in different fields of mathematics and applied sciences. For references, we mention just well-known general books on inequalities [1-4] and very informative and concise the specialized ones of Dragomir [5] and Steel [6], cf. also the survey [7].

P. Agarwal (凶)

Department of Mathematics, Anand International College of Engineering,

Near Kanota, Agra Road, Jaipur 303012, Rajasthan, India

e-mail: goyal.praveen2011@gmail.com

P. Agarwal

International Centre for Basic and Applied Sciences, Jaipur, India
A. A. Korenovskii
Department of Mathematical Analysis, Institute of Mathematics,
Economics and Mechanics, Odessa I.I. Mechnikov National University, Odessa, Ukraine
e-mail: anakor1958@gmail.com

\section{S. M. Sitnik}

Belgorod State National Research University, Belgorod, Russia

e-mail: sitnik@bsu.edu.ru 
The Cauchy-Bunyakovsky inequality for sums was found by Cauchy in 1821 [8] and for integrals by Bunyakovsky in 1859 [9]. It was rediscovered for integrals 26 years later in 1885 by Schwarz [10]. Different important applications derived from original text of Bunyakovsky's paper [9] were considered recently by J.Sándor in $[11,12]$. Inequality for inner product spaces oppositely to general opinion ascribed it to Schwarz was in fact first published only in 1932 by von Neumann in his book on mathematical foundations of quantum mechanics [13].

A new method for generalization of Cauchy-Bunyakovsky inequalities for finite sums, series and integrals was proposed by the third named author in early 1990s, these results are summed up in the survey [7], cf. also references to previous papers on this method in this survey. Shortly, an idea of this method is that every mean of two numbers defined by natural axioms leads to some classes of generalizations of Cauchy-Bunyakovsky inequalities.

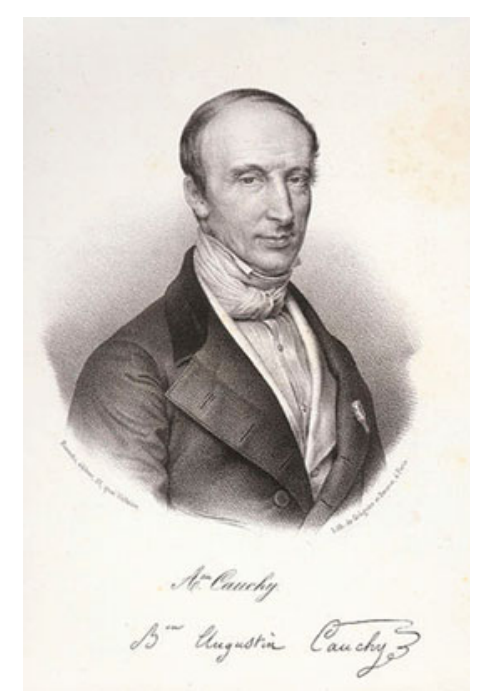

Augustin Louis Cauchy (1789-1857) 

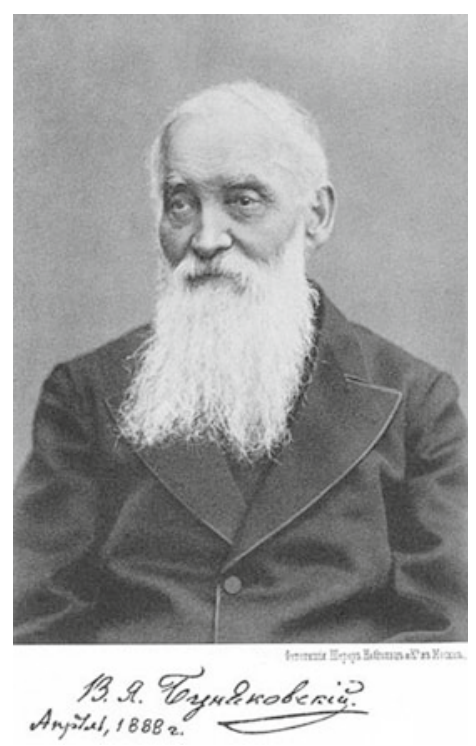

Viktor Yakovlevich Bunyakovsky (1804-1889)

\subsection{Means}

To demonstrate the results from [7] let us remind well-known definitions for arithmetic, geometric, quadratic and harmonic means for two positive numbers $x>0, y>0$ :

$$
\begin{gathered}
A(x, y)=\frac{x+y}{2}, G(x, y)=\sqrt{x, y}, Q(x, y)=\sqrt{\frac{x^{2}+y^{2}}{2}}, x, y \geq 0, \\
H(x, y)=A\left(\frac{1}{x}, \frac{1}{y}\right)=\frac{2 x y}{x+y}, x, y>0 .
\end{gathered}
$$

These classical means are:

(1) intermediate

$$
\min (x, y) \leq M(x, y) \leq \max (x, y),
$$

(2) homogenic

$$
M(\lambda x, \lambda y)=\lambda M(x, y), \lambda>0,
$$


(3) monotonic

$$
x_{2}>x_{1} \Rightarrow M\left(x_{2}, y\right)>M\left(x_{1}, y\right), y_{2}>y_{1} \Rightarrow M\left(x, y_{2}\right)>M\left(x, y_{1}\right) ;
$$

(4) symmetric

$$
M(x, y)=M(y, x) .
$$

For general mean, it is natural to define it as a function $M(x, y)$ for which all conditions (1)-(4) (or just some of them) are valid. This approach is not new and was used in many papers started from Cauchy.

From now to the end of the paper, we fix a condition for numbers in mean values $M(x, y)$ to be strictly positive, $x>0, y>0$.

We need also a notion of complementary mean.

Definition 1 For a mean $M(x, y)$ a complementary mean is defined by

$$
M^{*}(x, y)=\frac{x y}{M(x, y)}
$$

The most known classes of means are power means and Radó means. The power means are defined by cf. [1-3, 14, 15]

$$
\begin{aligned}
& M(x, y)=M_{\alpha}(x, y)=\left(\frac{x^{\alpha}+y^{\alpha}}{2}\right)^{\frac{1}{\alpha}},-\infty \leq \alpha \leq \infty, \alpha \neq 0 ; \\
& M_{-\infty}(x, y)=\min (x, y), M_{0}=\sqrt{x y}, M_{\infty}(x, y)=\max (x, y) .
\end{aligned}
$$

They form a parametric scale

$$
\alpha_{1}>\alpha_{2} \Rightarrow M_{\alpha_{1}}(x, y) \geq M_{\alpha_{2}}(x, y), \forall x, y .
$$

Three exceptional values $\alpha=-\infty, 0,+\infty$ are defined by limits.

So for classical means

$$
\begin{aligned}
& M_{-1}(x, y)=H(x, y) \leq M_{0}(x, y)=G(x, y) \\
& \leq M_{1}(x, y)=A(x, y) \leq M_{2}(x, y)=Q(x, y) .
\end{aligned}
$$

For power mean the complementary mean is

$$
\left(M_{\alpha}\right)^{*}=M_{-\alpha}
$$

Power means have many applications in different fields of mathematics and other sciences. And even two simplest arithmetical operations,$+ \times$ are expressed via them: 


$$
x+y=2 M_{1}(x, y), x \cdot y=\left(M_{0}(x, y)\right)^{2},
$$

the same is true for max and min values.

The second important class are the Radó means:

$$
\begin{gathered}
R_{\beta}(x, y)=\left(\frac{x^{\beta+1}-y^{\beta+1}}{(\beta+1)(x-y)}\right)^{\frac{1}{\beta}},-\infty \leq \beta \leq \infty, \beta \neq 0,-1 \\
R_{-\infty}(x, y)=\min (x, y), R_{\infty}(x, y)=\max (x, y)
\end{gathered}
$$

Obviously

$$
R_{-2}(x, y)=M_{0}(x, y), R_{1}(x, y)=M_{1}(x, y) .
$$

Exceptional values gives logarithmic mean

$$
R_{-1}(x, y)=L(x, y)=\frac{y-x}{\ln y-\ln x}
$$

and identric mean (the author prefer a name "multi-floored")

$$
R_{0}(x, y)=\frac{1}{e}\left(\frac{y^{y}}{x^{x}}\right)^{\frac{1}{y-x}} .
$$

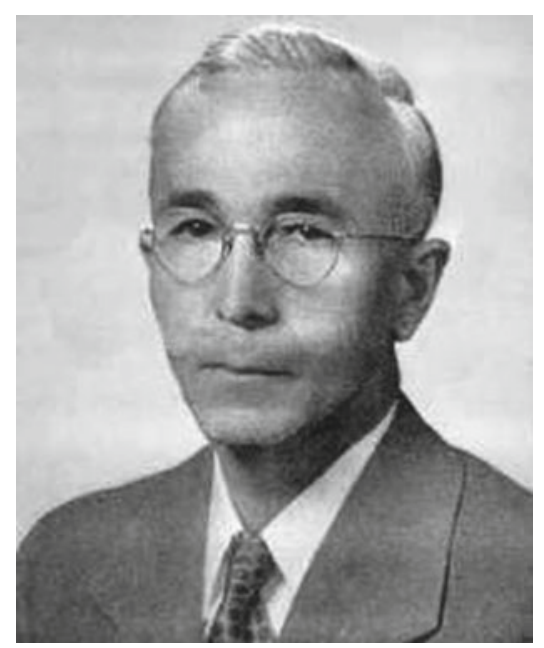

Tibor Radó (1895-1965)

The Radó means also form a parametric scale

$$
\beta_{1}>\beta_{2} \Rightarrow R_{\beta_{1}}(x, y) \geq R_{\beta_{2}}(x, y), \forall x, y ;
$$


with four exceptional values $\beta=\{-\infty,-1,0,+\infty\}$. The complementary mean now is

$$
R_{\beta}^{*}(x, y)=\frac{1}{R_{\beta}\left(\frac{1}{x}, \frac{1}{y}\right)} .
$$

The Radó means were introduced explicitly and studied by Tibor Radó in 1935 in [16], cf. also [3, 7]. Radó applied these means in his study of subharmonic functions. He proved important and non-trivial results on comparing of these means and power means. His results may be formulated in two main theorems.

Theorem R1 (T. Radó, 1935). Radó and power means coincides only for five values of parameters:

$$
M_{-\infty}=R_{-\infty}, M_{0}=R_{-2}, M_{\frac{1}{2}}=R_{\frac{1}{2}}, M_{1}=R_{1}, M_{\infty}=R_{\infty}
$$

So the most popular classical means are in both scales.

$H$ is another important result was a finding of parameter sets $(\alpha, \beta)$ for which the next inequalities are valid:

$$
M_{\alpha_{1}} \leq R_{\beta} \leq M_{\alpha_{2}}, R_{\beta_{1}} \leq M_{\alpha} \leq R_{\beta_{2}}
$$

Theorem R2 (T. Radó, 1935). The next best possible two-sided inequalities are valid for Radó means via power means:

$$
\begin{gathered}
M_{\frac{\alpha+2}{3}} \leq R_{\alpha} \leq M_{0}, \text { for } \alpha \in(-\infty,-2], \\
M_{0} \leq R_{\alpha} \leq M_{\frac{\alpha+2}{3}}, \text { for } \alpha \in[-2,-1], \\
M_{\frac{\alpha \ln 2}{\ln (1+\alpha)}} \leq R_{\alpha} \leq M_{\frac{\alpha+2}{3}}, \text { for } \alpha \in(-1,-1 / 2], \\
M_{\frac{\alpha+2}{3}} \leq R_{\alpha} \leq M_{\frac{\alpha \ln 2}{\ln (1+\alpha)}}, \text { for } \alpha \in[-1 / 2,1),
\end{gathered}
$$

(for $\alpha=0$ the above inequality is understood in the limiting sense $M_{\frac{2}{3}} \leq R_{0} \leq M_{\ln 2}$ ),

$$
M_{\frac{\alpha \ln 2}{\ln (1+\alpha)}} \leq R_{\alpha} \leq M_{\frac{\alpha+2}{3}}, \text { for } \alpha \in[1, \infty]
$$

Note that all indices in Theorem R2 are sharp and cannot be improved.

As spectacular consequences of T. Radó inequalities from Theorem R2 two folklore inequalities follow. First, it follows that beside the well-known inequality for logarithmic mean $M_{0} \leq L \leq M_{1}$, which is as old as published by V. Bunyakovsky in his seminal work [9], the next inequality is valid

$$
M_{0}(x, y)=\sqrt{x y} \leq L(x, y)=\frac{x-y}{\ln x-\ln y}
$$




$$
\leq M_{\frac{1}{3}}(x, y)=\left(\frac{x^{\frac{1}{3}}+y^{\frac{1}{3}}}{2}\right)^{3},
$$

and mean indices $0 ? 1 / 3$ are the best ones. Also for identric mean Theorem R2 gives:

$$
\begin{gathered}
M_{\frac{2}{3}}(x, y)=\left(\frac{x^{\frac{2}{3}}+y^{\frac{2}{3}}}{2}\right)^{\frac{2}{3}} \leq R_{0}(x, y) \\
=\frac{1}{e}\left(\frac{y^{y}}{x^{x}}\right)^{\frac{1}{y-x}} \leq M_{\ln 2}(x, y)=\left(\frac{x^{\ln 2}+y^{\ln 2}}{2}\right)^{\frac{1}{\ln 2}},
\end{gathered}
$$

and again indices are the best possible. Note that the simple estimate $M_{0} \leq R_{0} \leq M_{1}$ was also published by Bunyakovsky in [9].

Tibor Radó was a prominent mathematician in many fields, and his results on inequalities are very important too. They were many times reopened by and attributed to other researchers.

The world of means is very large and rich. Among other means just mention:

(1) Gini means, introduced by Corradó Gini in 1938

$$
\begin{gathered}
G i_{u, v}(x, y)=\left(\frac{x^{u}+y^{u}}{x^{v}+y^{v}}\right)^{\frac{1}{u-v}}, u \neq v, u, v \in \mathbb{R}, \\
G i_{u, v}(x, y)=\exp \left(\frac{x^{u} \ln x+y^{u} \ln y}{x^{u}+y^{u}}\right), u=v \neq 0, \\
G i_{u, v}(x, y)=G(x, y), u=v=0 .
\end{gathered}
$$

(2) Special case of Gini means - Lehmer means

$$
L e_{u}(x, y)=\frac{x^{u+1}+y^{u+1}}{x^{u}+y^{u}}, u \in \mathbb{R} .
$$

(3) Quasi-arithmetic means for non-negative values $x=\left(x_{1}, x_{2}, \ldots, x_{n}\right)$ and weights $p=\left(p_{1}, p_{2}, \ldots, p_{n}\right)$

$$
K_{p}(x)=f^{-1}\left(\sum_{k=1}^{n} p_{k} f\left(x_{k}\right)\right), \sum_{k=1}^{n} p_{k}=1 .
$$

(4) Iterated means starting from values $x_{0}, y_{0}$ and defined by a pair of means $(M, N)$ and a limit process:

$$
x_{n+1}=M\left(x_{n}, y_{n}\right), y_{n+1}=N\left(x_{n}, y_{n}\right),
$$




$$
\mu\left(M, N \mid x_{0}, y_{0}\right)=\mu\left(x_{0}, y_{0}\right)=\lim _{n \rightarrow \infty} x_{n}=\lim _{n \rightarrow \infty} y_{n}
$$

The most celebrated of iterated means is of course Gauss AGM one for a choice $M=M_{1}, N=M_{0}$, it equals

$$
\mu\left(M, N \mid x_{0}, y_{0}\right)=\frac{\frac{\pi}{2} x_{0}}{K\left(\sqrt{1-\left(\frac{y_{0}}{x_{0}}\right)^{2}}\right)}, 0<y_{0}<x_{0}
$$

$K(x)$ - the complete Legendre elliptic integral of the first kind.

Now let us stop here. There are much more known means of different kinds. In the survey [7] even there are general theorems characterizing all possible means. Our aim is to demonstrate that there are many concrete examples for constructions using general means. Let consider a method of generalizing Cauchy-Bunyakovsky inequalities using any means.

\subsection{Means Method for Generalizations of Cauchy-Bunyakovsky Inequalities}

Before formulations of our results let fix the next conditions: all functions below are continuous and integrals are of Riemann type. Further such restrictions will be omitted.

Now let us list some main results from the survey [7].

Theorem 1 Let $M$ be any abstract mean for which above formulated properties (1)-(4) are fulfilled, $M^{*}$-its complimentary mean. Then the next generalization of Cauchy-Bunyakovsky inequality is valid

$$
\begin{gathered}
\left(\int_{a}^{b} f(x) g(x) d x\right)^{2} \leq \int_{a}^{b}(M(f, g))^{2} d x \cdot \int_{a}^{b}\left(M^{*}(f, g)\right)^{2} d x \leq \\
\leq \int_{a}^{b}(f(x))^{2} d x \cdot \int_{a}^{b}(g(x))^{2} d x
\end{gathered}
$$

So we may state that an integral analogue of sufficient part of Carlitz-DaykinEliezer theorem (CDE theorem) is valid reformulated via means and complimentary means as proposed in [7]. Suddenly enough the necessary part of this theorem as it was proved in [7] is not valid contrary to the discrete version. For the discrete version Carlitz-Daykin-Eliezer theorem is necessary and sufficient, cf. [5]. This is a difference of discrete and integral generalizations of Cauchy-Bunyakovsky inequalities.

Note that only RHS of the Theorem 1 is non-trivial inequality, the LHS is trivial and being Cauchy-Bunyakovsky inequality itself. 
Now a choice of any known means and its complimentary ones generates by the Theorem 1 a number of generalizations of the Cauchy-Bunyakovsky LHS inequality. For example a choice of power means leads to the next

Theorem 2 For any positive functions $f(x), g(x), x \in[a, b]$, the next generalization of the Cauchy-Bunyakovsky inequality holds:

$$
\begin{aligned}
& \left(\int_{a}^{b} f(x) g(x) d x\right)^{2} \leq \int_{a}^{b}\left[M_{\alpha}(f, g)\right]^{2} d x \cdot \int_{a}^{b}\left[M_{-\alpha}(f, g)\right]^{2} d x= \\
= & \int_{a}^{b}\left(f^{\alpha}+g^{\alpha}\right)^{2 / \alpha} d x \cdot \int_{a}^{b} f^{2} g^{2}\left(f^{\alpha}+g^{\alpha}\right)^{-2 / \alpha} d x \leq \int_{a}^{b} f^{2} d x \cdot \int_{a}^{b} g^{2} d x .
\end{aligned}
$$

Consider special cases.

$$
\begin{array}{r}
\left(\int_{a}^{b} f(x) g(x) d x\right)^{2} \leq \int_{a}^{b}(\sqrt{f(x)}+\sqrt{g(x)})^{4} d x . \\
\cdot \int_{a}^{b} f^{2} g^{2} /(\sqrt{f(x)}+\sqrt{g(x)})^{4} d x \leq \int_{a}^{b} f^{2}(x) d x \cdot \int_{a}^{b} g^{2}(x) d x, \\
\left(\int_{a}^{b} f(x) g(x) d x\right)^{2} \leq \int_{a}^{b}(f(x)+g(x))^{2} d x . \\
\int_{a}^{b} f^{2} g^{2} /(f(x)+g(x))^{2} d x \leq \int_{a}^{b} f^{2}(x) d x \cdot \int_{a}^{b} g^{2}(x) d x, \\
\left(\int_{a}^{b} f(x) g(x) d x\right)^{b} \int_{a}^{b}\left(f^{2}(x)+g^{2}(x)\right) d x . \\
\int_{a}^{b} f^{2} g^{2} /\left(f^{2}(x)+g^{2}(x)\right), d x \leq \int_{a}^{b} f^{2}(x) d x \cdot \int_{a}^{b} g^{2}(x) d x .
\end{array}
$$

The case $\alpha=2$ is an integral variant of Milne inequality, cf. [1, 5, 7]. A choice of Radó means leads to the next

Theorem 3 Fornon-negative continuous functions $f(x), g(x), f(x), g(x) \neq 0, x \in$ $[a, b]$, the next generalization of the Cauchy-Bunyakovsky inequality holds:

$$
\begin{aligned}
\left(\int_{a}^{b} f(x) g(x) d x\right)^{2} & \leq \int_{a}^{b}\left[R_{\beta}(f, g)\right]^{2} d x \cdot \int_{a}^{b}\left[\frac{1}{R_{\beta}\left(\frac{1}{f}, \frac{1}{g}\right)}\right]^{2} d x \\
& \leq \int_{a}^{b} f^{2}(x) d x \int_{a}^{b} g^{2}(x) d x
\end{aligned}
$$

Special cases: 


$$
\begin{gathered}
\left(\int_{a}^{b} f(x) g(x) d x\right)^{2} \leq \int_{a}^{b}\left(\frac{f-g}{\ln \frac{f}{g}}\right)^{2} d x \cdot \int_{a}^{b} f^{2} g^{2} /\left(\frac{f-g}{\ln \frac{f}{g}}\right)^{2} d x \\
\leq \int_{a}^{b} f^{2}(x) d x \cdot \int_{a}^{b} g^{2}(x) d x \\
\left(\int_{a}^{b} f(x) g(x) d x\right)^{2} \leq \int_{a}^{b}\left[\frac{f^{f}}{g^{g}}\right]^{\frac{2}{f-g}} d x \cdot \int_{a}^{b} f^{2} g^{2} /\left(\frac{f^{f}}{g^{g}}\right)^{\frac{2}{f-g}} d x \\
\leq \int_{a}^{b} f^{2}(x) d x \cdot \int_{a}^{b} g^{2}(x) d x \\
\left(\int_{a}^{b} f(x) g(x) d x\right)^{2} \leq \int_{a}^{b}\left(f^{2}+f g+g^{2}\right) d x \cdot \int_{a}^{b} \frac{f^{2} g^{2}}{f^{2}+f g+g^{2}} d x \\
\leq \int_{a}^{b} f^{2}(x) d x \cdot \int_{a}^{b} g^{2}(x) d x .
\end{gathered}
$$

A choice of AGM mean leads to the next wonderful inequality

$$
\begin{gathered}
\left(\int_{a}^{b} f(x) g(x) d x\right)^{2} \leq \int_{a}^{b}\left[\frac{\max (f, g)}{K\left(\sqrt{1-\left(\frac{\min (f, g)}{\max (f, g)}\right)^{2}}\right)}\right]^{2} d x . \\
\cdot \int_{a}^{b}(\min (f, g))^{2}\left(K\left(\sqrt{1-\left(\frac{\min (f, g)}{\max (f, g)}\right)^{2}}\right)\right)^{2} d x \leq \int_{a}^{b} f^{2} d x \int_{a}^{b} g^{2} d x,
\end{gathered}
$$

$K(x)$ - the complete Legendre elliptic integral of the first kind. In the last inequality, arbitrary functions are arguments of a concrete special function - the complete Legendre elliptic integral of the first kind!

\section{Generalization of Cauchy-Bunyakovsky Inequality with Max-Min Values}

Now let consider the central inequality of this paper. It is a consequence of Theorem 1 (or of Theorem 2) for a choice $\alpha=+\infty$.

In this case, a mean is a maximum and its complimentary mean is a minimum.

Theorem 4 Let functions $f(x, y), g(x, y)$ be nonnegative on $[a, b]$. Then the next generalization of Cauchy-Bunyakovsky inequality holds

$$
\left(\int_{a}^{b} f(x) g(x) d x\right)^{2} \leq \int_{a}^{b}[\max (f, g)]^{2} d x \cdot \int_{a}^{b}[\min (f, g)]^{2} d x \leq
$$




$$
\leq \int_{a}^{b} f^{2}(x) d x \cdot \int_{a}^{b} g^{2}(x) d x . \quad(* * *)
$$

From the first glance, there is a chance that all three parts in Theorem 4 are just equal. But it is not the case. For example for functions $f(x)=x, g(x)=1-x$ and limits $a=0, b=1$ it reduces to the numerical inequality $\frac{1}{36}<\frac{7}{144}<\frac{1}{9}$.

It is hard to believe — but the simple inequality from Theorem 4 is new, it was somehow missed by researches in inequalities. It was first published in 1995 by S.M.Sitnik as a special case of the Theorem 1 above, cf. [7] and references to earlier 1990s papers therein. In turn, it would be interesting to prove directly this surprising result. Here we give three direct proofs of the mentioned inequality independently of Theorems 1 and 2.

Further in the paper, we consider real-valued continuous Riemann-integrable functions. These restrictions may be weaker, but it needs careful special considerations.

\subsection{Proofs of Min-Max Inequality}

First proof. It is an application of above Theorems 1 or 2 . This is an original indirect proof of 1995, included with proper references in [7].

\section{Second proof.}

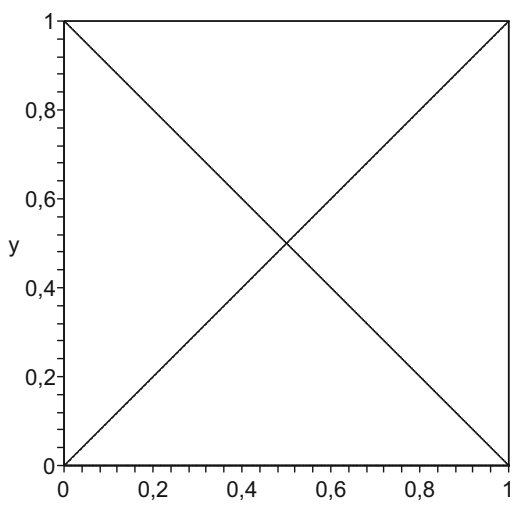

Example of "envelope" $f(x), g(x)=1-x, a=0, b=1$

Let introduce functions

$$
\begin{gathered}
E_{1}=\{x: f(x) \geq g(x)\}, E_{2}=\{x: f(x)<g(x)\} \\
p_{i}=\int_{E_{i}} f(x) d x, q_{i}=\int_{E_{i}} g(x) d x, i=1,2 .
\end{gathered}
$$


It follows

$$
\int_{E_{1}}(f(x)-g(x)) d x \cdot \int_{E_{2}}(f(x)-g(x)) d x \leq 0 .
$$

Now

$$
\begin{gathered}
\int_{E_{1}}(f(x)-g(x)) d x \cdot \int_{E_{2}}(f(x)-g(x)) d x \leq 0 \Leftrightarrow \\
\Leftrightarrow\left(p_{1}-q_{1}\right)\left(p_{2}-q_{2}\right) \leq 0 \Leftrightarrow\left(p_{1}+q_{2}\right)\left(p_{2}+q_{1}\right) \leq\left(p_{1}+p_{2}\right)\left(q_{1}+q_{2}\right) \Leftrightarrow \\
\Leftrightarrow \int_{a}^{b}[\max (f, g)] d x \cdot \int_{a}^{b}[\min (f, g)] d x \leq \int_{a}^{b} f(x) d x \cdot \int_{a}^{b} g(x) d x .
\end{gathered}
$$

For non-negative functions we may use equalities

$$
[\max (f, g)]^{2}=\max \left(f^{2}, g^{2}\right),[\min (f, g)]^{2}=\min \left(f^{2}, g^{2}\right),
$$

and so substituting in the last inequality $f, g$ to $f^{2}, g^{2}$ we derive the inequality (***) and prove the Theorem 4 .

\section{Third proof.}

It is well-known that the next formulas are valid for max and min as consequences of Vieta's theorem

$$
m=\frac{a+b-|a-b|}{2}, M=\frac{a+b+|a-b|}{2}, m=\min (a, b), M=\max (a, b) .
$$

So to prove $(* * *)$ denote

$$
I(f)=\int_{a}^{b} f(x) d x
$$

Then the LHS of (***) equals

$$
\begin{array}{r}
I\left((\min (f, g))^{2}\right) I\left((\max (f, g))^{2}\right) \\
=I\left(\left(\frac{f+g-|f-g|}{2}\right)^{2}\right) \cdot I\left(\left(\frac{f+g+|f-g|}{2}\right)^{2}\right) \\
=\frac{1}{16} I\left((f+g)^{2}-2(f+g)|f-g|+(f-g)^{2}\right) . \\
I\left((f+g)^{2}+2(f+g)|f-g|+(f-g)^{2}\right) \\
=\frac{1}{16} I\left(2 f^{2}+2 g^{2}-2(f+g)|f-g|\right) \cdot I\left(2 f^{2}+2 g^{2}+2(f+g)|f-g|\right) \\
=\frac{1}{4} I\left(f^{2}+g^{2}-(f+g)|f-g|\right) \cdot I\left(f^{2}+g^{2}+2(f+g)|f-g|\right) .
\end{array}
$$


After some obvious simplification it follows

$$
\frac{1}{4}\left[\left(I\left(f^{2}\right)\right)^{2}+\left(I\left(g^{2}\right)\right)^{2}-(I((f+g)|f-g|))^{2}+2 I\left(f^{2}\right) I\left(g^{2}\right)\right] .
$$

Then the difference of the RHS and LHS in (***) is represented as

$$
\begin{array}{r}
\text { RHS - LHS }=I\left(f^{2}\right) I\left(g^{2}\right) \\
-\frac{1}{4}\left[\left(I\left(f^{2}\right)\right)^{2}+\left(I\left(g^{2}\right)\right)^{2}-(I((f+g)|f-g|))^{2}+2 I\left(f^{2}\right) I\left(g^{2}\right)\right] \\
=\frac{1}{4}[I((f+g)|f-g|)]^{2}-\frac{1}{4}\left[I\left(f^{2}\right)-I\left(g^{2}\right)\right]^{2} \\
=\frac{1}{4} \cdot[I((f+g)|f-g|)+I((f+g)(f-g))] . \\
\cdot[I((f+g)|f-g|)-I((f+g)(f-g))] \\
=\frac{1}{4} \cdot I((f+g)((f-g)+|f-g|)) \cdot I((f+g)((f-g)-|f-g|)) \\
(f+g)(f-g) d x \cdot \int_{E(f \leq g)}(f+g)(g-f) d x \\
=\int_{E(f \geq g)}\left(f^{2}-g^{2}\right) d x \cdot \int_{E(f \leq g)}\left(g^{2}-f^{2}\right) d x .
\end{array}
$$

Obviously, the last expression is non-negative, and so the inequality (***) is proved.

Corollary 1 Let $f(x), g(x)$ be functions of ANY signs, not necessary positive. Then the next identity is valid for the difference of RHS and LHS of (***)

$$
\begin{aligned}
& \text { RHS of }(* * *)-\operatorname{LHS} \text { of }(* * *)= \\
& \int_{a}^{b} f^{2}(x) d x \cdot \int_{a}^{b} g^{2}(x) d x-\int_{a}^{b}[\max (f, g)]^{2} d x \cdot \int_{a}^{b}[\min (f, g)]^{2} d x= \\
& =\int_{E(f \geq g)}\left(f^{2}-g^{2}\right) d x \cdot \int_{E(f \leq g)}\left(g^{2}-f^{2}\right) d x .
\end{aligned}
$$

We emphasize that the last identity is valid for functions of arbitrary sign. So some generalizations of Cauchy-Bunyakovsky inequality of the form (***) are valid for functions of any sign.

Corollary 2 Let for functions $f(x), g(x)$ the condition $f(x)+g(x) \geq 0$ is fulfilled. Then the inequality-chain (***) holds true.

It follows from the above identity that if $f(x)+g(x) \leq 0$, then (***) is reversed, and equality conditions in (***) also follows. 


\section{Application to Inequalities for Special Functions with Mellin Transform Representations}

The inequality (***) from Theorem 4 may be applied to many special functions, especially represented as Mellin transform. Just as an example of such applications consider gamma and incomplete gamma functions, respectively [17]:

$$
\Gamma(a)=\int_{0}^{\infty} t^{a-1} e^{-t} d t, \Gamma(a, x)=\int_{x}^{\infty} t^{a-1} e^{-t} d t, \gamma(a, x)=\int_{0}^{x} t^{a-1} e^{-t} d t,
$$

Now, specify in $(* * *)$ :

$$
f(x)=x^{\frac{a+1}{2}} e^{-\frac{x}{2}}, g(x)=x^{\frac{a-1}{2}} e^{-\frac{x}{2}}, a>0 .
$$

It follows that

$$
\max (f, g)=\left\{\begin{array}{l}
f(x), x \geq 1, \\
g(x), x \leq 1,
\end{array} \quad \min (f, g)=\left\{\begin{array}{l}
g(x), x \geq 1 \\
f(x), x \leq 1
\end{array}\right.\right.
$$

So by $(* * *)$ we infer

$$
\begin{aligned}
\Gamma^{2}(a+1) \leq(\gamma(a+1,1)+\Gamma(a-1,1)) \cdot(\gamma(a-1,1) & +\Gamma(a+1,1)) \leq \\
& \leq \Gamma(a+2) \cdot \Gamma(a)
\end{aligned}
$$

which is a Turán type inequality with respect to the argument $a, \mathrm{cf}$. [18-21]. Therefore the following result is proved.

Theorem 5 Let $a>0$, then the above inequality for gamma and incomplete gamma functions is valid.

Note that this is a stronger result than log-convexity of classical gamma function. It may be combined with known results on log-convexity of some special functions $[18,19]$ to derive new inequalities. In fact, the above inequality in the same way may be proved for any functions represented as Mellin transform.

\section{Concluding Remarks and Bibliography Comments}

The above proofs of refinements of Cauchy-Bunyakovsky inequalities may be also applied in more general settings: infinite domains of integration, Lebesgue integrals, multivariate functions and its domains. But accurate proofs for such generalizations are not always direct and easy.

Our results are also generalized to integral Minkowski and discrete CauchyBunyakovsky and Minkowski inequalities. 
It seems that before publications in 1990s summed up in the survey [7] inequality (***) from Theorem 4 was never published. But for recent years it became popular and was reopened not once and also generalized in interesting directions. Let us mention some of them.

In 2013 the inequality (***) was reopened in [22], the proofs are based on an equality like Corollary 1 to Theorem 4 . Starting from the discrete case the author went to integral case by limits. For the discrete case, an interesting combinatorial proof is proposed. The author also considered some examples to demonstrate nontriviality of $(* * *)$, but instead of our 'envelope' piecewise constant functions are used. In this paper $(* * *)$ is considered in equivalent form

$$
\begin{aligned}
\left(\int_{a}^{b} f(x) g(x) d x\right)^{2} & \leq \int_{a}^{b}\left[\max \left(f^{2}, g^{2}\right)\right] d x \cdot \int_{a}^{b}\left[\min \left(f^{2}, g^{2}\right)\right] d x \\
& \leq \int_{a}^{b} f^{2}(x) d x \cdot \int_{a}^{b} g^{2}(x) d x,
\end{aligned}
$$

as for positive functions

$$
\max \left(f^{2}, g^{2}\right)=(\max (f, g))^{2}, \min \left(f^{2}, g^{2}\right)=(\min (f, g))^{2} .
$$

Here, input functions $f, g, f(x)+g(x) \geq 0$ also can be considered in the manner exposed previously.

In interesting papers of Pinelis [23, 24] also inequality (***) was considered with proper references to the original result in [7]. The author proved an intriguing result, that generalizations of the form (***) exist only for the Cauchy-Bunyakovsky inequality and do not exist for Rogers-Hölder-Riesz inequality. Exactly it is proved that refinements to Rogers-Hölder-Riesz inequality of the form

$$
\begin{aligned}
\int_{a}^{b} f(x) g(x) d x & \leq\left(\int_{a}^{b}(\max (f, g))^{p} d x\right)^{\frac{1}{p}} \cdot\left(\int_{a}^{b}(\min (f, g))^{q} d x\right)^{\frac{1}{q}} \\
& \leq\left(\int_{a}^{b} f^{p}(x) d x\right)^{\frac{1}{p}} \cdot\left(\int_{a}^{b} g^{q}(x) d x\right)^{\frac{1}{q}}
\end{aligned}
$$

does not hold for $p>1,1 / p+1 / q=1$, except for $p=q=2$ which turns out to be the Cauchy-Bunyakovsky inequality's interpolation. Moreover, those refinements do not hold in the dual form

$$
\begin{aligned}
\int_{a}^{b} f(x) g(x) d x & \leq\left(\int_{a}^{b}(\max (f, g))^{q} d x\right)^{\frac{1}{q}} \cdot\left(\int_{a}^{b}(\min (f, g))^{p} d x\right)^{\frac{1}{p}} \\
\leq & \left(\int_{a}^{b} f^{p}(x) d x\right)^{\frac{1}{p}} \cdot\left(\int_{a}^{b} g^{q}(x) d x\right)^{\frac{1}{q}}
\end{aligned}
$$


And more, even a compound of last two inequalities in the weakest form do not hold, it means that the next inequality do not hold

$$
\begin{gathered}
\min \left\{\left(\int_{a}^{b}(\max (f, g))^{p} d x\right)^{\frac{1}{p}} \cdot\left(\int_{a}^{b}(\min (f, g))^{q} d x\right)^{\frac{1}{q}},\right. \\
\left.\left(\int_{a}^{b}(\max (f, g))^{q} d x\right)^{\frac{1}{q}} \cdot\left(\int_{a}^{b}(\min (f, g))^{p} d x\right)^{\frac{1}{p}}\right\} \\
\leq \max \left\{\left(\int_{a}^{b} f^{p}(x) d x\right)^{\frac{1}{p}} \cdot\left(\int_{a}^{b} g^{q}(x) d x\right)^{\frac{1}{q}},\right. \\
\left.\left(\int_{a}^{b} f^{q}(x) d x\right)^{\frac{1}{q}} \cdot\left(\int_{a}^{b} g^{p}(x) d x\right)^{\frac{1}{p}}\right\}
\end{gathered}
$$

for some choice of $f(x), g(x)$. A counterexample is found in [23, 24] that even such minimized variant of LHS can be greater than maximized variant of RHS for Rogers-Hölder-Riesz inequality. It is worth to mention that in the case of Young inequality this kind of optimization is successfully used in [7].

So the case we consider in this paper of refinement of Cauchy-Bunyakovsky inequality in the form $(* * *)$ is exceptional! Due to it it is even more interesting.

The author in $[23,24]$ also considered generalizations not only with max and min values, but also with more general transformations. In turn, it seems that these are covered by the famous Carlitz-Daykin-Eliezer theorem (CDE theorem), (cf. [3, 5] for classical formulation and [7] for the formulation via means).

Mention also papers of 2015-2016 [25, 26] which do not contain original new results and are compiled of consequences of Carlitz-Daykin-Eliezer theorem (authors do not mention this theorem), known results from [7] and further trivial applications.

Another important connected line of results is the reverse Cauchy-Bunyakovsky inequality, including Schweitzer, Kantorovich, Pólya-Szegó, Shisha-Mond, DiazMetcalf, Rennie and similar inequalities, see e.g. [5, 27-29].

\section{References}

1. G.H. Hardy, Littlewood, G. Pólya, Inequalities. Cambridge Mathematical Library, 2nd edn. (Cambridge University Press, Cambridge, 1952), 324 p

2. E.F. Beckenbach, R. Bellman, Inequalities. Second revised printing. Ergebnisse der Mathematik und ihrer Grenzgebiete. Neue Folge, Band 30.-(Springer, New York, Inc., 1965), xi+198 p

3. D.S. Mitrinović, J.E. Pečarić, A.M. Fink, Classical and New Inequalities in Analysis (Kluwer, 1993), $740 \mathrm{p}$ 
4. A.W. Marshall, I. Olkin, B.C. Arnold, Inequalities: Theory of Majorization and Its Applications, 2nd edn. (Springer, 2011), $909 \mathrm{p}$

5. S.S. Dragomir, A Survey on Cauchy-Buniakowsky-Schwartz Type Discrete Inequalities. RGMIA monographs, (2003), $214 \mathrm{p}$

6. J.M. Steele, The Cauchy-Schwarz Master Class: An Introduction to the Art of Mathematical Inequalities. (Cambridge University Press, Cambridge, 2004), $306 \mathrm{p}$

7. S.M. Sitnik, Generalized Young and Cauchy-Bunyakowsky Inequalities with Applications: A Survey 2010, (2012). arXiv:1012.3864, $51 \mathrm{p}$

8. A.L. Cauchy, Cours d'analyse de l'École Royale Polytechnique. I partie. Analyse algebrique. (Oeuvres complè tes, II serie, III). (Paris, 1821)

9. V. Buniakowski, Sur quelques inégalités concernant les intégrales ordinaires et les intégrales aux différences finies Mémoires de l' Acad. de St. - Pétersbourg (VII), 9 (1859)

10. H.A. Schwarz, Ueber ein Flachen Kleinsten Flacheninhalts betreffendes Problem der Variationsrechnung Acta. Soc. Sci. Fenn. 15 (1885), pp. 315-362

11. J. Sandór, Applications of the Cauchy-Bouniakowsky Inequality in the Theory of Means. RGMIA Research Report Collection, 17, Article 11, 18 p (2014)

12. J. Sandór, Bouniakowsky and the Logarithmic Mean Inequalities. RGMIA Research Report Collection, 17, Article 5, 5 p (2014)

13. A. Pietsch, History of Banach Spaces and Linear Operators. Birkhäuser, 877 p (2007)

14. P.S. Bullen, D.S. Mitrinović, P.M. Vasić, Means and Their Inequalities, 2nd edn. 2015 (D.Reidel Publishing Company, Dordrecht, 1988), $480 \mathrm{p}$

15. P.S. Bullen, Handbook of Means and Their Inequalities (Kluwer, 2003), $587 \mathrm{p}$

16. T. Radó, On convex functions. Trans. Amer. Math. Soc. 37, 266-285 (1935)

17. F.W.J. Ed. Olver, NIST Handbook of Mathematical Functions (Cambridge University Press, Cambridge, 2010), $968 \mathrm{p}$

18. D.B. Karp, S.M. Sitnik, Log-convexity and log-concavity of hypergeometric-like functions. J. Math. Anal. Appl. 364(2), 384-394 (2010)

19. D.B. Karp, S.M. Sitnik, Inequalities and monotonicity of ratios for generalized hypergeometric function. J. Approximation Theory 161, 337-352 (2009)

20. S.M. Sitnik, K.h. Mehrez, Proofs of some conjectures on monotonicity of ratios of Kummer, Gauss and generalized hypergeometric functions. Analysis (De Gruyter) 36(4), 263-268 (2016)

21. Kh. Mehrez, S.M. Sitnik, Results in mathematics (Springer International Publishing). Funct. Inequalities Mittag-Leffler Funct. 72(1-2), 703-714 (2017)

22. J.X. Xiang, A note on the Cauchy-Schwarz inequality. Am. Math. Monthly 120(5), 456-459 (2013)

23. I. Pinelis, On the Hölder and Cauchy-Schwarz Inequalities, 3 p (2015). arXiv:1503.00348v2

24. I. Pinelis, On the Hölder and Cauchy-Schwarz inequalities. Am. Math. Monthly 121, 1 (2015)

25. E. Omey, On Xiang's observations concerning the Cauchy-Schwarz inequality. Am. Math. Monthly 122(7), 696-698 (2015)

26. M. Masjed-Jamei, E. Omey, Improvement of some classical inequalities. J. Inequalities Spec. Funct. 7(1), 18-28 (2016)

27. G. Alpargu, The Kantorovich Inequality, with Some Extensions and with Some Statistical Applications (Thesis, McGill University, Montréale, Canada, 1996)

28. N.S. Barnett, S.S. Dragomir, An additive reverse of the Cauchy-Bunyakovsky-Schwarz integral inequality. Appl. Math. Lett. 21(4), 388-393 (2008)

29. T.K. Pogány, A new (probabilistic) proof of the Diaz-Metcalf and Pólya-Szegó inequalities and some of their consequences. Theory Probab. Math. Statist. 70, 113-122 (2005) 